\title{
Dependence of the electronic and transport properties of metal-MoSe interfaces on contact structures
}

\author{
Deniz Çakır ${ }^{*}$ and F. M. Peeters ${ }^{\dagger}$ \\ Department of Physics, University of Antwerp, Groenenborgerlaan 171, 2020 Antwerp, Belgium \\ (Received 27 February 2014; revised manuscript received 13 May 2014; published 4 June 2014)
}

\begin{abstract}
Transition metal dichalcogenides (TMDs) are considered as promising candidates for next generation of electronic and optoelectronic devices. To make use of these materials, for instance in field effect transistor applications, it is mandatory to know the detailed properties of contacts of such TMDs with metal electrodes. Here, we investigate the role of the contact structure on the electronic and transport properties of metal-MoSe $\mathrm{S}_{2}$ interfaces. Two different contact types, namely face and edge contacts, are studied. We consider both low (Sc) and high $(\mathrm{Au})$ work function metals in order to thoroughly elucidate the role of the metal work function and the type of metal. First principles plane wave calculations and transport calculations based on nonequilibrium Green's function formalism reveal that the contact type has a large impact on the electronic and transport properties of metal-MoSe $e_{2}$ interfaces. For the Sc electrode, the Schottky barrier heights are around $0.25 \mathrm{eV}$ for face contact and bigger than $0.6 \mathrm{eV}$ for edge contact. For the Au case, we calculate very similar barrier heights for both contact types with an average value of $0.5 \mathrm{eV}$. Furthermore, while the face contact is found to be highly advantageous as compared to the edge contact for the Sc electrode, the latter contact becomes a better choice for the Au electrode. Our findings provide guidelines for the fabrication of TMD-based devices.
\end{abstract}

DOI: 10.1103/PhysRevB.89.245403

PACS number(s): 73.22.-f, 73.63.-b

\section{INTRODUCTION}

After the successful isolation of graphene in 2004, twodimensional (2D) materials have attracted a lot of attention [1-4]. Among them, transition metal dichalcogenides (TMDs) are promising materials due to their distinctive electronic and optical properties [5-13]. Unlike graphene, the electronic properties of TMDs are diverse, ranging from semiconductor such as $\mathrm{MoS}_{2}$ to metal such as $\mathrm{NbS}_{2}$. The absence of a band gap in graphene makes it difficult to use in field effect transistors (FETs), for instance. To overcome this band gap problem, especially, the Mo- and W-based TMDs having band gaps ranging from the visible to the near infrared are considered as ideal candidates.

Although $\mathrm{MoS}_{2}$ and $\mathrm{MoSe}_{2}$ monolayers have direct band gaps of 1.86 and $1.55 \mathrm{eV}$, respectively, the mobility of the charge carrier is very low in these 2D materials as compared to graphene, which has a superior carrier velocity that is only 300 times smaller than the speed of light. However, using high dielectric materials as substrates in, e.g., field effect transistors made of TMDs were found to significantly increase the mobility from 184 to about $700 \mathrm{~cm}^{2} /$ Vs due to the suppression of Coulomb scattering [14]. In a recent study, a $\mathrm{MoSe}_{2}$ based FET was shown [15] to be $n$-type and possess a high gate modulation, with a current on/off ratio larger than $10^{6}$. The room temperature mobility was measured as large as $50 \mathrm{~cm}^{2} /(\mathrm{Vs})$ and exhibits a strong temperature dependence. As in the case of $\mathrm{MoS}_{2}$-based FETs, using a more suitable dielectric material can enhance the carrier mobility in $\mathrm{MoSe}_{2}$-based FETs.

The right choice of the metal contact in the fabrication of an electronic device is a key step towards low contact

\footnotetext{
*deniz.cakir@uantwerpen.be

†francois.peeters@uantwerpen.be
}

resistances, which is necessary to reduce power loss and to improve carrier injection efficiency across the interface. Low (high) work function metals are generally chosen to achieve small $n$-( $p$-)type Schottky barriers. The barrier height for electron tunneling across the interface and the nature of the contact between a TMD and an electrode were shown to tune significantly by changing the type of the electrode [14,16-19]. Moreover, the experimental and first-principles studies on $\mathrm{Al} / \mathrm{Ag}$ contacts with $\mathrm{WSe}_{2}$ suggested that metals with $d$ orbitals can help in forming ohmic contacts to monolayer $\mathrm{WSe}_{2}$ [17]. One key bottleneck is the achievement of ohmic contacts for TMDs to improve the on-state mode of the FETs. Thus it is essential to get a detailed understanding of the contacts between metals and TMDs for the fabrication of next generation electronic devices.

In this work we choose Sc as metal contact which is mentioned by the fact that in a recent experimental work it was found that Sc forms better contacts with thin $\mathrm{MoS}_{2}$ flakes, resulting in a lower contact resistance and high carrier injection as compared to $\mathrm{Ti}, \mathrm{Ni}$, and $\mathrm{Pt}$ [14]. Due to much smaller lattice mismatch with Sc $(\sim 0.5 \%)$, we consider $\mathrm{MoSe}_{2}$ instead of $\mathrm{MoS}_{2}$. The lattice mismatch is an important parameter that should be minimized to get a favorable interface geometry and a better bonding in order to maximize the orbital overlap between metal and TMD atoms. Computationally, to fit a $\mathrm{MoS}_{2}$ monolayer on a Sc (0001) surface, we need much larger unit cells. In order to thoroughly elucidate the role of the metal work function and the type of metal, we also consider $\mathrm{Au}$ contact which has a much higher work function and different electronic properties. In this study, we find that different contact structures, namely edge and face contacts, at the metal-TMD interfaces result in quite different electronic and transport properties. In addition, the type of metal electrode also plays a key role in determining the interface electronic properties. While the face contact structure is a better choice for the Sc electrode, the edge contact becomes a more suitable 
contact type for the Au case. Due to very similar electronic properties of $\mathrm{MoSe}_{2}$ and $\mathrm{MoS}_{2}$, we believe that our findings are also valid for $\mathrm{MoS}_{2}$ and will help to design low resistance contacts.

\section{METHODS}

First we optimize the structure of the different $\mathrm{Sc}-\mathrm{MoSe}_{2}$ interfaces including face and edge contacts denoted in Fig. 1, using density functional theory (DFT) at the generalized gradient approximation (GGA/PBE) [20] level, as implemented in the VASP code $[21,22]$. From an experimental point of view, one may argue that an edge contact is not a realistic contact structure. However, previously it was found that when $\mathrm{Ti}$ and $\mathrm{Pd}$ was deposited on a graphene flake, the spontaneous formation of a metal-graphene edge contact occurred as a result of the metal-graphene reaction instead of a simple carbon diffusion process [23]. Therefore, the present work provides a detailed understanding that will help in the design of efficient contacts between metal substrates and TMDs. In edge contact configuration, we simulate a $\mathrm{MoSe}_{2}$ monolayer with an average length of $20 \AA$ along the $z$ direction, which is also the transport direction in our electron transport calculations. This length scale is sufficient to eliminate artificial contact-contact interaction due to the finite size effect. In $x$ and $y$ directions, we use periodic boundary conditions to represent an infinite $\mathrm{MoSe}_{2}$ monolayer. Similar to graphene, finite size TMDs can have two different terminations, namely zigzag and armchair. To investigate the effect of termination, both zigzag and armchair terminations are considered. The interface is modeled using a rectangular Sc (0001) surface unit cell to fit $\mathrm{MoSe}_{2}$ with a tiny lattice mismatch. The uppermost two Sc atomic layers and the $\mathrm{MoSe}_{2}$ monolayer are allowed to relax. We use the projector augmented wave (PAW) method [24], a plane wave kinetic energy cutoff of $400 \mathrm{eV}$ and a regular k-point grid with a spacing of $0.02 \AA^{-1}$ for the Brillouin zone sampling [25]. The convergence is achieved when the difference of the total energies between two consecutive ionic steps is less than $10^{-5} \mathrm{eV}$ and the maximum force allowed on each atom is set to be $0.01 \mathrm{eV} / \AA$. The interlayer spacing (which is measured between two planes formed by the nearest Se atoms on the adjacent monolayers) in the edge contact configuration is taken to be larger than $6.5 \AA$, and therefore we approach the monolayer limit. At this interlayer separation, the electronic properties (such as the size and nature of the band gap) of $\mathrm{MoSe}_{2}$ almost resembles the isolated single layer $\mathrm{MoSe}_{2}$. Therefore, our supercell dimensions are enough to study the electronic and transport properties of the $\mathrm{MoSe}_{2}$ monolayer contacted to the $\mathrm{Sc}(0001)$ surface. To prevent spurious interaction between the periodic images, a large vacuum spacing (at least $12 \AA$ ) is introduced.

Electronic transport across the $\mathrm{Sc}-\mathrm{MoSe}_{2}$ interface is calculated using the self-consistent nonequilibrium Green's functions (NEGF) technique as implemented in TranSIESTA [26], which is interfaced with the SIESTA code [27]. Single- $\zeta$ (plus polarization) numerical orbital basis sets are used for all atoms. In a recent theoretical study, it was shown that single- and double- $\zeta$ polarized basis sets yield very similar results for metal/TMDs junctions [28]. We employ normconserving pseudopotentials [29], the GGA/PBE functional, and an energy cutoff for the real-space mesh of $150 \mathrm{Ry}$. While the SIESTA code uses a localized basis set and norm-conserving pseudopotentials, the calculated lattice parameters for the $\mathrm{MoSe}_{2}$ monolayer and bulk agree very well with those obtained from the VASP code. For instance, the lattice parameter for the isolated single layer $\mathrm{MoSe}_{2}$ is found to be $3.32 \AA$ with VASP and (a)

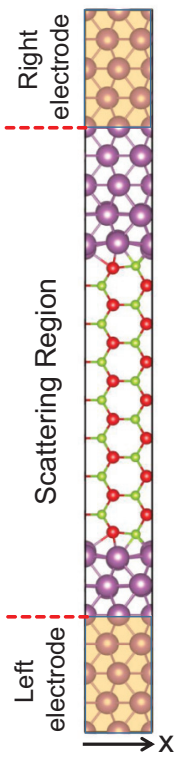

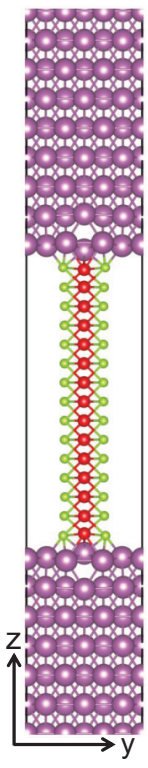

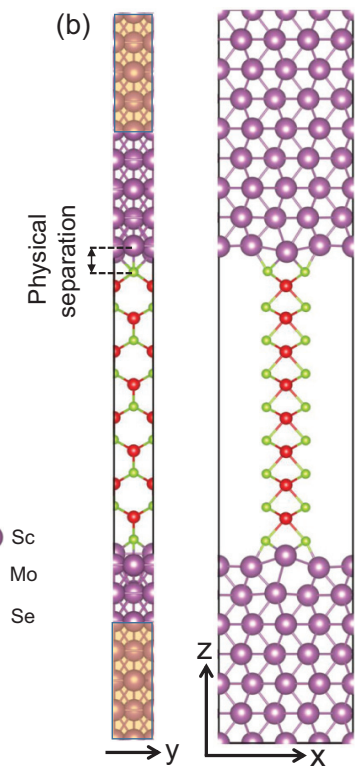

(c)

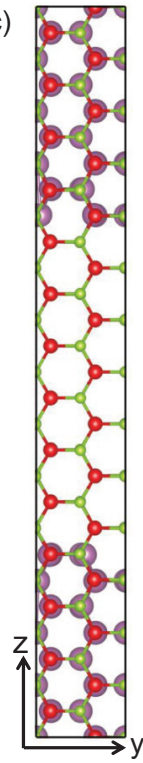

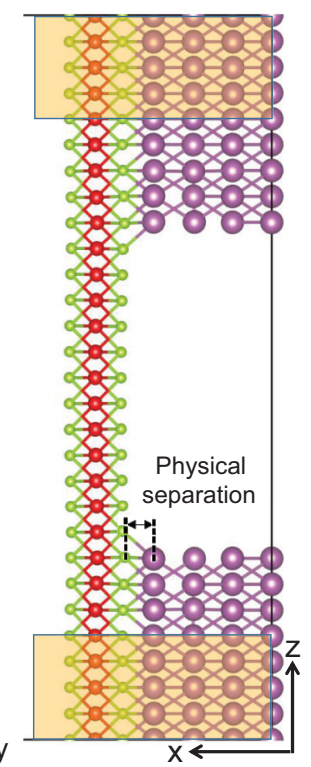

FIG. 1. (Color online) Optimized lowest energy structures of the $\mathrm{MoSe}_{2}$ monolayer contacted to the $\mathrm{Sc}(0001)$ surface from different views used in the electronic structure and transport calculations. In (a) edge contact with zigzag termination, in (b) edge contact with armchair termination, and face contact in (c). The physical separation is the distance between the topmost layer of the metal electrode and topmost layer of the $\mathrm{MoSe}_{2}$ monolayer at the interface. The $z$ direction is the transport direction in the TranSIESTA calculations. Shaded parts show the electrode regions. Figures are prepared using the VESTA program [32]. 
3.36 A with SIESTA. Similarly, the calculated lattice parameters for bulk $\mathrm{MoSe}_{2}$ are 3.31 (3.37) and 13.00 (13.15) Å with VASP (SIESTA), in good agreement with the experimental values of 3.28 and $12.95 \AA$ [30]. In order to get accurate transmission spectrum and current as a function of applied bias, the 2D Brillouin zone normal to the transport direction (which is the $z$ direction in this study) is sampled by the $k$ meshes of $50 \times 29$ for the edge contact with armchair termination, $20 \times 60$ for the edge contact with zigzag termination, and $1 \times 100$ for the face contact configuration.

\section{STRUCTURAL AND ELECTRONIC PROPERTIES OF Sc-MoSe $\mathrm{S}_{2}$ INTERFACES}

The optimized structures with the minimum total energy are shown in Fig. 1. The lattice mismatch between the pristine Sc surface and $\mathrm{MoSe}_{2}$ monolayer is at most $\sim 0.5 \%$. To further minimize it, we use a rectangular unit cell with the lattice parameters given by the average of the lattice parameters of the isolated $\mathrm{MoSe}_{2}$ monolayer and the pristine metal electrode. The corresponding lattice parameters in the plane normal to the $z$ direction are set to $5.73 \AA$ and $9.94 \AA$ for the edge contact with armchair termination and $11.47 \AA$ and $3.31 \AA$ for the edge contact with zigzag termination; see Figs. 1(a) and 1(b). Thickness of the Sc slab normal to the $z$ direction in the edge contact geometry is at least $16.1 \AA$, corresponding to seven Sc layers, four of which constitute the electrode region. For the face contact geometry shown in Fig. 1(c), the lattice parameter in the $y$ direction is $5.73 \AA$. The length of the $\mathrm{MoSe}_{2}$ monolayer contacted to the Sc surface in the face contact is at least $11.55 \AA$. In addition, we use a four layers slab for the face contact configuration and the vacuum region in the $x$ direction is fixed to $12 \AA$. If one compares the relaxed structures of face and edge contact configurations, the edge contact leads to more structural relaxation of the Sc surface relative to the face contact case. The physical separation, defined in Fig. 1 as the distance between the topmost layer of the metal electrode and the topmost layer of the $\mathrm{MoSe}_{2}$ monolayer at the interface, is a measure of the interaction strength between the metal surface and the $\mathrm{MoSe}_{2}$ monolayer. While the physical separation is $2.01 \AA$ in face contact, it becomes $1.76 \AA$ in edge contact. Similarly, it was reported that edge contact results in a much smaller physical separation for $\mathrm{MoS}_{2}$ and $\mathrm{WSe}_{2}$ on a wide range of metal substrates ranging from low work function such as Ti to high work function metals such as Pd [19]. The Sc-Se interatomic distances are $2.68-2.73 \AA$ in edge contact and $2.75-2.79 \AA$ in face contact. In both contact geometries, Se atoms prefer to occupy the hollow site of the Sc (0001) surface. Similar to Se, the Mo atom (which directly binds to the Sc surface) resides slightly away from the hollow site in edge contact. To compare the binding strengths for both contact types, we define the binding energy between the metal and the $\mathrm{MoSe}_{2}$ layer as the total energy difference between the combined $\left(\mathrm{Sc} / \mathrm{MoSe}_{2}\right)$ and the isolated systems (pristine Sc surface and $\mathrm{MoSe}_{2}$ monolayer). Since the edge contact contains unsaturated selenium atoms as compared to saturated and rather inert selenium atoms in the face contact, the binding of $\mathrm{MoSe}_{2}$ to the Sc surface in face contact is considerably weaker than that in edge contact, with the binding energy per

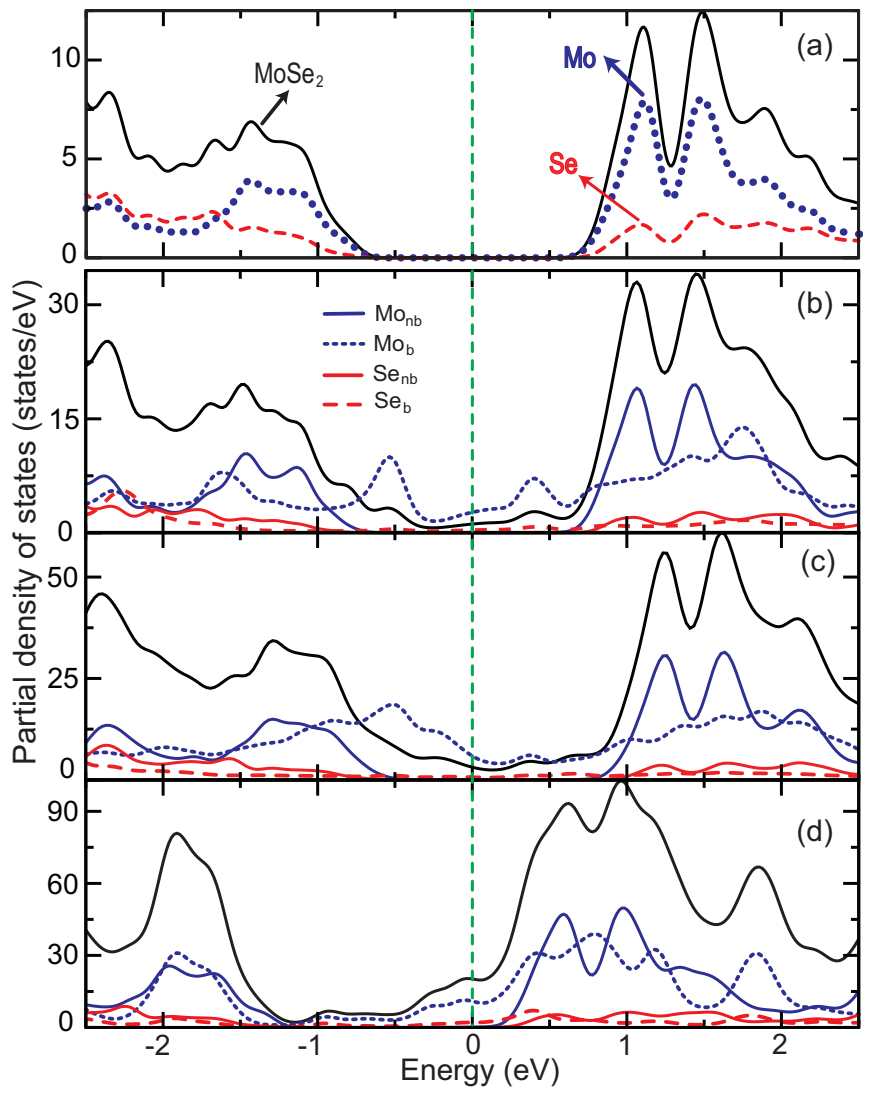

FIG. 2. (Color online) Partial density of states of (a) free standing $\mathrm{MoSe}_{2}$, (b) edge contacted $\mathrm{MoSe}_{2}$ with zigzag termination, (c) armchair termination, and (d) face contacted $\mathrm{MoSe}_{2}$, as calculated with VASP. Vertical dashed line denotes the Fermi level. In (a) black solid, blue dotted, and red dashed curves show the projection of the total DOS on $\mathrm{MoSe}_{2}, \mathrm{Mo}$, and Se, respectively. In (b), (c), and (d) we plot projection of the total DOS on different Mo and Se atoms. Here, $\mathrm{Mo}_{n b}$ (blue line) and $\mathrm{Mo}_{b}$ (dashed blue line) are used to show the molybdenum atoms far away from interface and at the interface, respectively. Similarly, $\mathrm{Se}_{n b}$ (red line) and $\mathrm{Se}_{b}$ (dashed red line) are the selenium atoms far away from interface and at the interface, respectively. The PDOS of the $\mathrm{Mo}_{n b}, \mathrm{Mo}_{b}, \mathrm{Se}_{n b}$, and $\mathrm{Se}_{b}$ atoms is enlarged in order to make them visible.

surface metal atom of $1.26 \mathrm{eV}$ in the case of edge contact as compared to $0.93 \mathrm{eV}$ in face contact.

We next investigated the electronic structure of the $\mathrm{MoSe}_{2} / \mathrm{Sc}$ interfaces by calculating the partial density of states (PDOS) of adsorbed $\mathrm{MoSe}_{2}$. While DFT within the GGA/PBE has a tendency to misestimate the band gap for most semiconductors, the PBE functional fairly predicts the band gap of $\mathrm{MoSe}_{2}$ with a band gap value of $1.46 \mathrm{eV}$, in close agreement with the experimental gap of $1.55 \mathrm{eV}$. Consistent with the VASP calculation and the experiment, the SIESTA code predicts a band gap value of $1.53 \mathrm{eV}$. Previously, semilocal functionals were successfully employed for TMDs/metal interfaces [14,16-19]. This clearly implies that our computational setup is adequate to study $\mathrm{MoSe}_{2} / \mathrm{Sc}$ interfaces. Figure 2 displays PDOS of edge and face contacted $\mathrm{Sc} / \mathrm{MoSe}_{2}$ interfaces. We also calculated the PDOS of free standing $\mathrm{MoSe}_{2}$ for comparison. Although the strong interaction leads to a remarkable hybridization of 
metal and $\mathrm{MoSe}_{2}$ states to a certain extent, we can still identify the valence and conduction bands of adsorbed $\mathrm{MoSe}_{2}$ in both edge and face contact configurations. Interestingly, in spite of the stronger $\mathrm{MoSe}_{2}-\mathrm{Sc}$ interaction, PDOS in the edge contact case is quite similar to that for pristine $\mathrm{MoSe}_{2}$ monolayer. In edge contact only the small portion of $\mathrm{MoSe}_{2}$ (only a single $\mathrm{MoSe}_{2}$ unit) binds to the Sc surface as compared to face contact, thereby leading to a small effect on the electronic properties of pristine $\mathrm{MoSe}_{2}$ monolayer. Due to the strong interaction with the Sc surface, the electronic properties of the $\mathrm{MoSe}_{2}$ monolayer is markedly modified in the face contact geometry such that bonding to the Sc surface removes the degeneracies, broadens the states, and introduces localized states that altogether significantly broadens the local density of states in the conduction band region.

The most striking difference is that edge and face contacts result in different electronic structures at the interfaces. In edge contact configuration, Fermi level lies within the band gap of $\mathrm{MoSe}_{2}$, resulting in the formation of a Schottky barrier at the interface. The calculated Schottky barrier height (which is the difference between the conduction band edge and the Fermi level) for electrons in armchair termination is about $0.75 \mathrm{eV}$. Another crucial point is that the $\mathrm{MoSe}_{2} / \mathrm{Sc}$ interface in face contact configuration displays a much higher density of states at the Fermi level than that in edge contact, shown in Fig. 2(d). Since Sc is a low work function transition metal with a value of $3.5 \mathrm{eV}$, it may lead to $n$-type doping of TMDs. For the face contact configuration, the Fermi level is found to be very close to the conduction band of $\mathrm{MoSe}_{2}$, resulting in $n$-type doping of $\mathrm{MoSe}_{2}$. This finding is consistent with the experimental results that predicted $n$-type characteristics of $\mathrm{MoS}_{2}$ based FETs. Moreover, it was indicated that the metal/MoS 2 interface is strongly affected by Fermi level pinning close to the conduction band minimum of $\mathrm{MoS}_{2}$, in agreement with our results. In a recent theoretical study, $\mathrm{Ti}$ is found to be the best face contact for $\mathrm{MoS}_{2}$ monolayer and is an attractive $n$-type contact metal, verifying our findings for Sc. According to PDOS calculations, the states near the Fermi level mainly originate from the Mo atoms with a small contribution from the Se atoms. Figure 2 also shows the PDOS depending on the position of the Mo and $\mathrm{Se}$ atoms on the $\mathrm{MoSe}_{2}$ monolayer. While the $\mathrm{Mo}_{b}$ and $\mathrm{Se}_{b}$ atoms sit at the interface and have a direct interaction with the Sc surface, the $\mathrm{Mo}_{n b}$ and $\mathrm{Se}_{n b}$ atoms reside in the nonbonded part of the $\mathrm{MoSe}_{2}$ monolayer and are far away from the interface. A reduction in the wave function overlap between the $\mathrm{Mo}_{b}$ and $\mathrm{Se}_{b}$ atoms owing to change in bond lengths as a result of strong interaction with the surface atoms results in a spreading of the Mo $d$ states into band gap region of the $\mathrm{MoSe}_{2}$ monolayer. Adversely, the orbitals of $\mathrm{Mo}_{n b}$ and $\mathrm{Se}_{n b}$ atoms do not spillover the band gap region and can be considered as unperturbed. These results clearly suggest that the band gap states are mainly localized at the interface. In a recent study, it was shown that such band gap states are the origin of the Fermi level pinning at the metal-TMD interfaces [31].

In face contact, the Fermi level is shifted upwards, to about $0.2 \mathrm{eV}$ below the bottom of the $\mathrm{MoSe}_{2}$ conduction band. The high density of states at the Fermi level may be a sign of a low resistance ohmic contact for the face contact configuration. Additionally, in order to realize a ohmic contact, the states at and near the Fermi level should be delocalized allowing

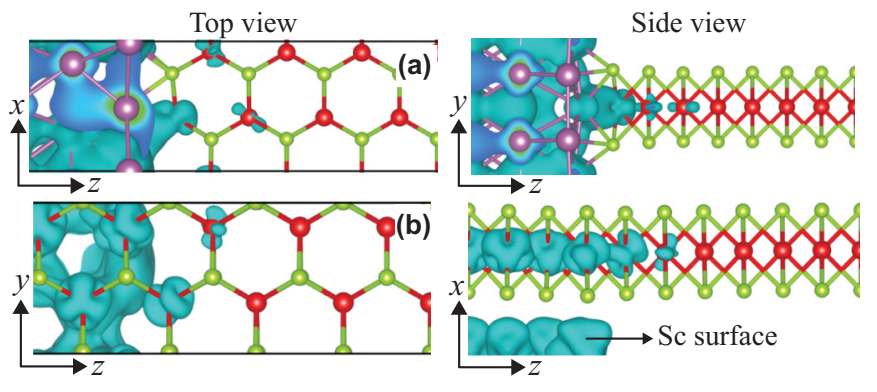

FIG. 3. (Color online) Top and side views of the energy resolved charge densities for (a) edge contact with armchair termination and (b) face contact. The charge densities associated with states in the energy range of $E_{F}-0.25 \mathrm{eV}<E<E_{F}+0.25 \mathrm{eV}$. Here, $E_{F}$ is the Fermi level. The value of isosurface is $0.005 e / \AA^{3}$. The charge density is superimposed to the ball-and-stick model. Figures are prepared using the VESTA program [32].

electron transfer across the metal-TMD interface with a low or negligible barrier. To test this, we calculated the electron density for states around the Fermi level (i.e., within the energy range of $E_{F}-0.25 \mathrm{eV}<E<E_{F}+0.25 \mathrm{eV}$, where $E_{F}$ is the Fermi level) depicted in Fig. 3. A careful analysis revealed that the high density of states around the Fermi level is mostly localized at the interface and does not extend to the nonbonded part of $\mathrm{MoSe}_{2}$. Since the Se atoms at the interface have a very small contribution to the PDOS as compared to the Mo atoms within this energy range, the main contribution to the charge density originates from the Mo and electrode atoms, which may enhance the contact resistance for the face contact. Similarly, the density of states around the Fermi level is just localized at the interface in the edge contact geometry. The existence of a Schottky barrier and the localization of the charge density at the interface give rise to higher contact resistance. In a recent experimental work, the insertion of a thin $\mathrm{TiO}_{2}$ tunnel barrier between the Co electrode and $\mathrm{MoS}_{2}$ was demonstrated to reduce the Schottky barrier significantly and hence the resistance of the contacts by alleviation of the Fermi level pinning [33].

It is now useful to compare the electronic properties of $\mathrm{Sc} / \mathrm{MoSe}_{2}$ interfaces with those of $\mathrm{Au} / \mathrm{MoSe}_{2}$. We use exactly the same device geometries, denoted in Fig. 1, for Au electrode but with different lattice parameters since $\mathrm{Au}$ and Sc have different crystal structures and lattice constants. The lattice parameters for the edge contact are $5.77 \AA$ in the $x$ direction and $9.99 \AA$ in the $y$ direction. For the face contact geometry, the lattice parameter in $y$ direction is set to $5.77 \AA$ and we use a three layers slab. Unlike Sc, Au has completely filled $d$ orbitals and partially occupied $s$ orbital, which may lead to totally different electronic properties when it is used as a substrate. Another difference is that $\mathrm{Au}$ is a high work function metal with a value of $5.2 \mathrm{eV}$. Figure 4 shows the PDOS of edge and face contact structures for Au. Similar to the finding of Popov et al. for the $\mathrm{MoS}_{2} / \mathrm{Au}$ interface [18], $\mathrm{MoSe}_{2}$ does not strongly interact with the Au surface especially in the face contact case with an equilibrium binding distance of $3.2 \AA$. Since $\mathrm{Au}$ is a large work function transition metal, we found that the $\mathrm{Au}$ electrode leads to $p$-type doping of $\mathrm{MoSe}_{2}$, i.e., the Fermi level appears close to the valence band of $\mathrm{MoSe}_{2}$. In contrast to Sc, 


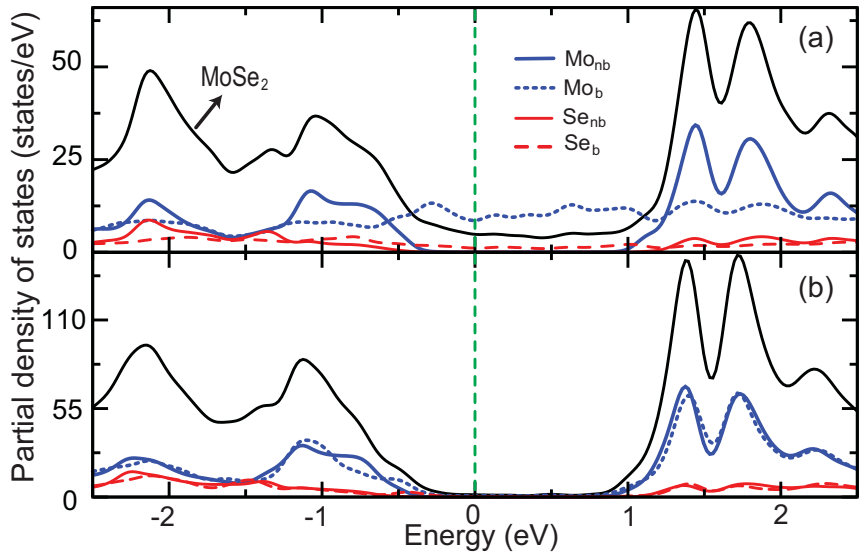

FIG. 4. (Color online) Partial density of states of (a) the edge contacted $\mathrm{MoSe}_{2}$ with zigzag termination and (b) the face contacted $\mathrm{MoSe}_{2}$ for the Au electrode, as calculated with VASP. Vertical dashed line denotes the Fermi level. We plot the projection of the total DOS on the different Mo and Se atoms. Here, $\mathrm{Mo}_{n b}$ (blue line) and $\mathrm{Mo}_{b}$ (dashed blue line) are used to show the molybdenum atoms far away from interface and at the interface, respectively. Similarly, $\mathrm{Se}_{n b}$ (red line) and $\mathrm{Se}_{b}$ (dashed red line) are the selenium atoms far away from interface and at the interface, respectively. The PDOS of the $\mathrm{Mo}_{n b}$, $\mathrm{Mo}_{b}, \mathrm{Se}_{n b}$, and $\mathrm{Se}_{b}$ atoms is enlarged in order to make them visible.

the electronic properties of the edge contact configuration in the Au case seems to be more advantageous as compared to the face contact owing to relatively high density of states near the Fermi level. However, the position of the Fermi level with respect to the valence band maximum (VBM) is almost the same, leading to a similar Schottky barrier height for holes in both contact types. Our calculations suggest that not only contact type but also the type of metal has a strong effect on the electronic properties of the metal/TMD interfaces.

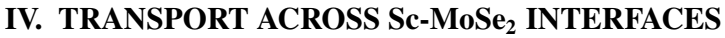

In the previous part, we find that a face contact is better than an edge contact for the Sc electrode since there is a high density of states near the Fermi level in the former contact. In addition to this, we reveal that the states near the Fermi level are not delocalized over the whole $\mathrm{MoSe}_{2}$ monolayer. After this point, an important question arises: how do these states behave in electron transport across the interface? In this part, we investigated the electron transport properties of $\mathrm{MoSe}_{2}$ monolayer contacted to the Sc electrode. The structures of the $\mathrm{Sc}-\mathrm{MoSe}_{2}$ junctions studied in this work are shown in Fig. 1. In order to calculate the electron transport in a $\mathrm{Sc} / \mathrm{MoSe}_{2}$ junction, the structures shown in Fig. 1 are partitioned into three regions: left electrode, right electrode, and scattering region. The electrodes are modeled as semi-infinite. The scattering region has to be large enough to avoid interaction between the electrodes. The length of the $\mathrm{MoSe}_{2}$ monolayer in the scattering region is larger than $19 \AA$, which is high enough to eliminate electrode-electrode interaction. In an electron transport calculation, transmission coefficient $T(E)$ is the most important quantity that measures the energydependent total probability of electrons to propagate through a device. The transmission coefficient $\langle T(E)\rangle$ averaged over a

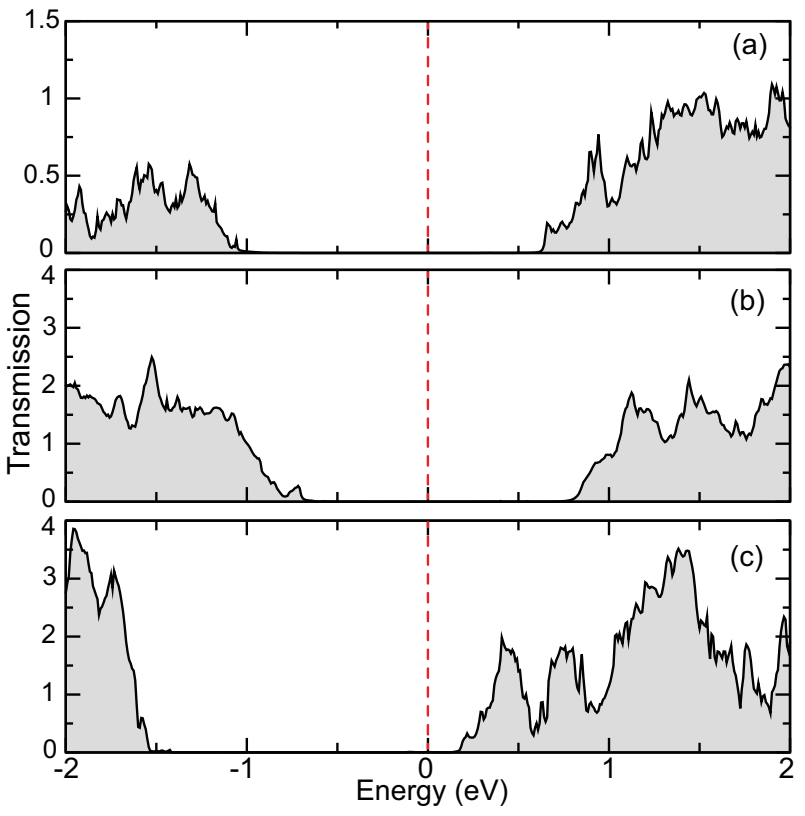

FIG. 5. (Color online) Transmission for edge contact (a) in zigzag termination, (b) in armchair termination, and (c) face contact at zero bias, as calculated with TransIESTA. The zero of energy is at the Fermi level $E_{F}$ indicated by the vertical dashed lines.

two-dimensional Brillouin zone perpendicular to the transport direction is defined as $\langle T(E)\rangle=\operatorname{Tr}\left[G^{r} \Gamma_{L}(E) G^{a}(E) \Gamma_{R}(E)\right]$. Here, $G^{r(a)}$ is the retarded (advanced) Green function and $\Gamma_{(L, R)}(E)=i\left(\Sigma_{L, R}^{r}-\Sigma_{L, R}^{a}\right)$ describes the level broadening due to left electrode and right electrodes expressed in terms of the electrode self-energies $\Sigma_{L, R}$, which describe the influence of the electrodes on the scattering region.

At low bias, the states at and near the Fermi level are expected to determine the electron transport across the interface. Figure 5 shows zero bias transmission for both edge and face contact configurations. The peaks in the transmission spectrum clearly correspond to the valence band (VB) and the conduction band (CB) of the adsorbed $\mathrm{MoSe}_{2}$. A band gap with a value of $1.6 \mathrm{eV}$ (which is close to the band gap of the pristine $\mathrm{MoSe}_{2}$ ) separates VB and CB. Consistent with the PDOS calculations, the Fermi level in the face contact appears very close to $\mathrm{CB}$ maximum. Moreover, the states at the Fermi level lead to very small transmission $\left[\sim 0.003 G_{0}\right.$, where $G_{0}$ $\left(=2 e^{2} / h\right)$ is the unit of conductance]. Although, according to PDOS calculations, there is a high density of states at the Fermi level in the face contact case, the electron transport across the $\mathrm{MoSe}_{2} / \mathrm{Sc}$ contact is mainly of a tunneling nature at zero bias, confirming that the states at and near the Fermi level are localized at the $\mathrm{Sc} / \mathrm{MoSe}_{2}$ interface. The Schottky barrier heights for both edge and face contacts calculated from their corresponding transmission spectrums as the difference of the Fermi level and the bottom of conduction band minimum are consistent with those calculated from PDOS calculations. Comparison of the transmission spectrum of the armchair and zigzag terminations of the edge contact reveals that the armchair edge contact is more conductive than the zigzag edge contact. As seen in Fig. 2, the valence and conduction bands of both the pristine and adsorbed $\mathrm{MoSe}_{2}$ monolayer are 
mainly composed of the Mo $d$ orbitals with a relatively smaller contribution from Se $p$ orbitals. In the edge contact with zigzag termination, the Mo atoms have either no direct or a weaker interaction with surface atoms, resulting in a smaller orbital overlap between the Mo and surface atoms, and consequently leading to a smaller transmission as compared to the armchair termination. Adversely, in a recent theoretical study, it was shown that the electron transport along the armchair and zigzag directions of the infinite $\mathrm{MoS}_{2}$ monolayer are almost the same [34]. These results show that the detailed binding structure at the interface is also quite crucial. Consistent with the PDOS plots shown in Figs. 2(b) and 2(c), the Fermi level appears within the band gap of the $\mathrm{MoSe}_{2}$ monolayer.

Bai et al. studied the effect of the contact area and binding distance on electron transport across the metal/MoSe 2 interfaces [28]. The authors stressed that strong binding does not assure a transparent interface for carriers. There are other factors such as contact area, contact type, and binding distance between TMD and metal electrode that play a significant role in the electronic and the carrier transport properties of $\mathrm{TMD} / \mathrm{metal}$ interfaces, in agreement with the present work. We expect that our findings can be generalized to other contacts between TMDs and metals. The present work and previous studies suggest that other low (high) work function metals such as $\mathrm{Ti}$ (Ag) exhibit electronic and charge transport properties similar to Sc $(\mathrm{Au})$. With the right metal and certain contact geometry, it is possible to get low contact resistances at the $\mathrm{TMD} / \mathrm{metal}$ interfaces.

Finally, the current across the $\mathrm{Sc} / \mathrm{MoSe}_{2}$ interfaces is calculated using the Landauer-Buttiker formula

$$
I=\frac{2 e}{h} \int\langle T(E, V)\rangle\left[f\left(E-\mu_{L}\right)-f\left(E-\mu_{R}\right)\right] d E,
$$

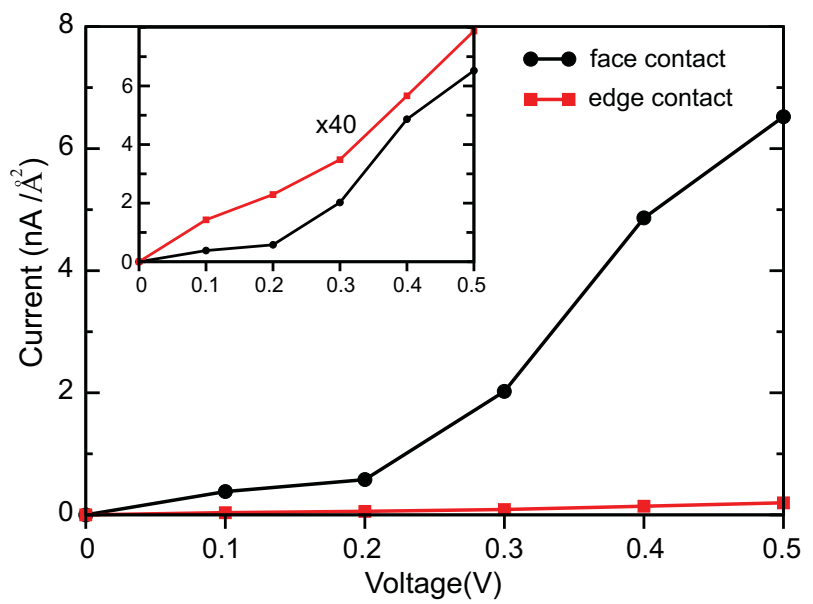

FIG. 6. (Color online) Current per total area covered by $\mathrm{MoSe}_{2}$ monolayer on the Sc surface in the device region as a function of voltage for face contact (filled black circles) and edge contact with armchair termination (filled red squares). The area of the $\mathrm{MoSe}_{2}$ monolayer in the device region directly contacted to the Sc surface in the face (edge) contact geometry is 28.65 (20.74) $\AA^{2}$. In the inset, to compare the current of edge contact with that of face contact on a similar scale we multiple it with 40 . where $f(E)(=1 /[\exp (E / k T)+1])$ is the Fermi distribution function and $\mu_{L / R}$ is the chemical potential in the left or right electrode. $V=\left(\mu_{L}-\mu_{R}\right) / e$ defines the bias window. In order to apply an external bias, the Fermi level of the electrodes are shifted relative to each other and the electronic occupations of the system are determined by the electrochemical potential (i.e., $\mu_{L / R}$ ) of the electrodes. The calculated current-voltage $(I-V)$ curves for face and edge contacts are shown in Fig. 6. While current mildly varies up to $0.2 \mathrm{~V}$, it starts to increase more rapidly beyond this voltage in face contact. When voltage exceeds $0.2 \mathrm{~V}$, the conduction band of $\mathrm{MoSe}_{2}$ starts to enter the bias window, leading to a noticeable increase in the current. Comparing the $I-V$ relation of both contact types, the face contact structure gives rise to larger current values than edge contact within the same voltage range. Since, edge contact has a much larger Schottky barrier with a barrier hight of $0.75 \mathrm{eV}$, the calculated $I-V$ relation is relatively constant. To make a reliable comparison between edge and face contacts, similar lengths in both contact types were considered for the free standing (i.e., nonbonded) part of the $\mathrm{MoSe}_{2}$ monolayer.

\section{CONCLUSION}

The $\mathrm{Sc} / \mathrm{MoSe}_{2}$ system is investigated to demonstrate the dependence of the electronic properties and carrier transport on the contact type. The face contact is found to be highly advantageous as compared to the edge contact. The Fermi level appears just below the conduction band edge of $\mathrm{MoSe}_{2}$, leading to a much smaller Schottky barrier and hence smaller tunnel barrier in face contact. The calculated Schottky barrier heights are around $0.25 \mathrm{eV}$ for the face contact and bigger than $0.6 \mathrm{eV}$ for the edge contact. We also find that not only the contact type but also the type of metal electrode plays a key role on the electronic properties of the metal-TMD interfaces. In contrast to the Sc case, the edge contact appears to be more advantageous as compared to face contact in the case of $\mathrm{Au}$ electrode. Our findings clearly show that the carrier transport across a metal/TMD interface is mainly dominated by the detailed structural properties at the interfaces such that the Schottky barrier heights and charge distribution are largely controlled by the contact structure. To obtain a low contact resistance, not only the type of the metal and TMD but also the contact structure should be carefully considered.

\section{ACKNOWLEDGMENTS}

This work was supported by the Flemish Science Foundation (FWO-Vl) and the Methusalem foundation of the Flemish government. Computational resources were provided by TUBITAK ULAKBIM, High Performance and Grid Computing Center (TR-Grid e-Infrastructure), and HPC infrastructure of the University of Antwerp (CalcUA), a division of the Flemish Supercomputer Center (VSC), which is funded by the Hercules foundation. D.C. is supported by a FWO Pegasus-short Marie Curie Fellowship. 
[1] K. S. Novoselov, A. K. Geim, S. V. Morozov, D. Jiang, S. C. Dubonos, I. V. Grigorieva, and A. A. Firsov, Science 306, 666 (2004).

[2] A. K. Geim and K. S. Novoselov, Nat. Mater. 6, 183 (2007).

[3] A. H. C. Neto, F. Guinea, N. M. R. Peres, K. S. Novoselov, and A. K. Geim, Rev. Mod. Phys. 81, 109 (2009).

[4] S. D. Sarma, S. Adam, E. H. Hwang, and E. Rossi, Rev. Mod. Phys. 83, 407 (2011).

[5] K. F. Mak, C. Lee, J. Hone, J. Shan, and T. F. Heinz, Phys. Rev. Lett. 105, 136805 (2010).

[6] C. Ataca, H. Sahin, and S. Ciraci, J. Phys. Chem. C 116, 8983 (2012).

[7] Q. H. Wang, K. Kalantar-Zadeh, A. Kis, J. N. Coleman, and M. S. Strano, Nat. Nanotechnol. 7, 699 (2012).

[8] X. Song, J. Hu, and H. Zeng, J. Mater. Chem. C 1, 2952 (2013).

[9] M. Xu, T. Liang, M. Shi, and H. Chen, Chem. Rev. 113, 3766 (2013).

[10] X. Huang, Z. Zeng, and H. Zhang, Chem. Soc. Rev. 42, 1934 (2013).

[11] M. Chhowalla, H. S. Shin, G. Eda, L.-J. Li, K. P. Loh, and H. Zhang, Nat. Chem. 5, 263 (2013).

[12] S. Horzum, H. Sahin, S. Cahangirov, P. Cudazzo, A. Rubio, T. Serin, and F. M. Peeters, Phys. Rev. B 87, 125415 (2013).

[13] H. Sahin, S. Tongay, S. Horzum, W. Fan, J. Zhou, J. Li, J. Wu, and F. M. Peeters, Phys. Rev. B 87, 165409 (2013).

[14] S. Das, H.-Y. Chen, A. V. Penumatcha, and J. Appenzeller, Nano Lett. 13, 100 (2013).

[15] S. Larentis, B. Fallahazad, and E. Tutuc, Appl. Phys. Lett. 101, 223104 (2012).

[16] W. Chen, E. J. G. Santos, W. Zhu, E. Kaxiras, and Z. Zhang, Nano Lett. 13, 509 (2013).
[17] W. Liu, J. Kang, D. Sarkar, Y. Khatami, D. Jena, and K. Banerjee, Nano Lett. 13, 1983 (2013).

[18] I. Popov, G. Seifert, and D. Tománek, Phys. Rev. Lett. 108, 156802 (2012).

[19] J. Kang, D. Sarkar, W. Liu, D. Jena, and K. Banerjee, in IEEE International Conference on Electron Devices Meeting (IEDM) 2012, San Francisco, CA (IEEE, Piscataway, NJ, 2012), pp. 17.4.1-17.4.4.

[20] J. P. Perdew, K. Burke, and M. Ernzerhof, Phys. Rev. Lett. 77, 3865 (1996).

[21] G. Kresse and J. Hafner, Phys. Rev. B 47, 558 (1993).

[22] G. Kresse and J. Furthmuller, Phys. Rev. B 54, 11169 (1996).

[23] C. Gong, S. McDonnell, X. Qin, A. Azcatl, H. Dong, Y. J. Chabal, K. Cho, and R. M. Wallace, ACS Nano 8, 642 (2014).

[24] G. Kresse and D. Joubert, Phys. Rev. B 59, 1758 (1999).

[25] H. J. Monkhorst and J. D. Pack, Phys. Rev. B 13, 5188 (1976).

[26] M. Brandbyge, J.-L. Mozos, P. Ordejón, J. Taylor, and K. Stokbro, Phys. Rev. B 65, 165401 (2002).

[27] J. M. Soler, E. Artacho, J. D. Gale, A. Garcia, J. Junquera, P. Ordejón, and D. Sánchez-Portal, J. Phys.: Condens. Matter 14, 2745 (2002).

[28] Z. Bai, T. Markussen, and K. S. Thygesen, arXiv:1311.2393 [cond-mat.mes-hall].

[29] N. Troullier and J. L. Martins, Phys. Rev. B 43, 1993 (1991).

[30] J. Morales, J. Santos, and J. Tirado, Solid State Ion. 83, 57 (1996).

[31] C. Gong, L. Colombo, R. M. Wallace, and K. Cho, Nano Lett. 14, 1714 (2014).

[32] K. Momma and F. Izumi, J. Appl. Crystallogr. 41, 653 (2008).

[33] A. Dankert, L. Langouche, M. V. Kamalakar, and S. P. Dash, ACS Nano 8, 476 (2014).

[34] M. Ghorbani-Asl, S. Borini, A. Kuc, and T. Heine, Phys. Rev. B 87, 235434 (2013). 